\title{
Modeling and analysis of variable PPM for visible light communications
}

\author{
Jong-Ho Yoo and Sung-Yoon Jung*
}

\begin{abstract}
Variable pulse position modulation (VPPM) is a new modulation scheme that supports simultaneously illumination with dimming control and communication. In this paper, the transmitter, optical wireless channel, and receiver structure of VPPM were modeled mathematically, and their error performance for examining the communication performance according to dimming level and data rate was analyzed. The results highlight the need for careful consideration of the tradeoff between the dimming flexibility and communication performance according to the channel condition in VPPM.
\end{abstract}

\section{Introduction}

Recently, light-emitting diode (LED) research has focused on the emerging lighting infrastructure due to Green IT technical innovations. LED lighting is superior to existing incandescent and fluorescent lighting in terms of the long life expectancy, high tolerance to humidity, minimal heat generation, and low power consumption. Another important benefit of LEDs is that it is a controllable digital device. Therefore, many attempts have been made to merge LEDs with information technology (IT). Among them, visible light communication (VLC), which uses LED as a communicating device, has emerged as a new Green IT convergence technology [1-3]. Generally, VLC uses intensity modulation with a direct detection (IM/DD) scheme, which utilizes the amplitude (or intensity) of light to transmit data. Human eyes recognize only the mean intensity when light changes faster than the maximum flickering time period, which is defined as $5 \mathrm{~ms}$. Therefore, both lighting and communication can be implemented simultaneously. In IEEE, the corresponding VLC standardization was recently published by the IEEE Standards Association [4].

In the IEEE standard, the variable pulse position modulation (VPPM) scheme was proposed and adopted as one of the modulation schemes for VLC. To support illumination with dimming control and communication simultaneously, this scheme uses binary PPM for

\footnotetext{
* Correspondence: syjung@ynu.ac.kr

Department of Electronic Engineering, Yeungnam University, 214-1 Daedong, Gyeongsan-si, Gyeongsangbuk-do 712-749, Republic of Korea
}

communication and the pulse width for dimming control. Figure 1 shows an operating example of the VPPM. In Figure 1, the dimming and communication functions of the VPPM appear to operate independently. Although the VPPM concept is well described in the standard, no attempt has been made to model the structure of VPPM and analyze its ability to examine the performance tradeoff between the dimming and communication functions according to the channel condition. More efficient communication and lighting parameters for VPPM can be realized if its communication performance can analyzed according to the dimming level and channel environment.

This paper proposes the transmit-receive (TX-RX) modem structure, which is a mathematical model of VPPM and an optical wireless channel. The error performance of the model was then analyzed with regard to the dimming level for the illumination and channel condition. Based on the analytical and simulated results, this paper provides a guideline for the VPPM operation to satisfy both lighting and communication abilities for a given environmental condition.

\section{System description}

Figure 2 shows the proposed structure of the VLC system based on VPPM. When binary data $b \in\{0,1\}$ are transmitted, the VPPM modulated signal $s(t)$ is generated considering the target dimming level as follows: 


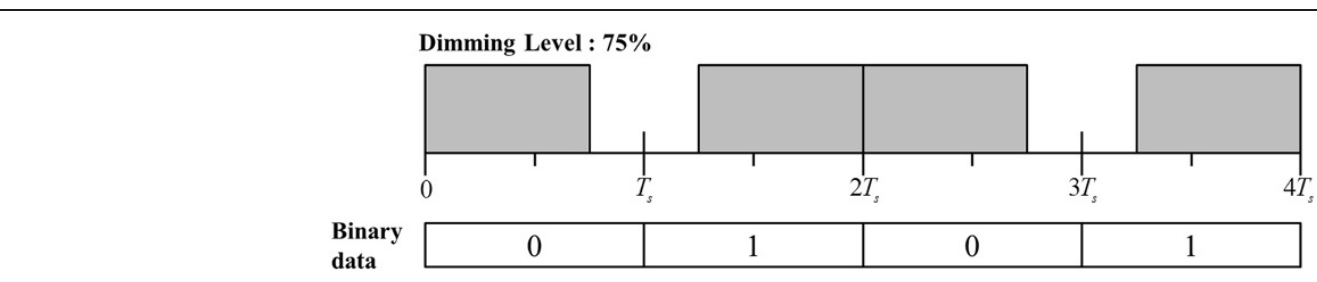

Figure 1 Operating example of VPPM in case of 75\% dimming level.

$$
s(t)=\left\{\begin{array}{l}
\sqrt{E_{\mathrm{s}} \cdot \frac{d}{50}} \cdot \phi_{0}(t), \text { for } b=0 \\
\sqrt{E_{\mathrm{s}} \cdot \frac{d}{50}} \cdot \phi_{1}(t), \text { for } b=1
\end{array},\right.
$$

where Es is the symbol energy, $\mathrm{d}$ is the dimming level $(0 \leq d \leq 100) . \phi_{i}(t)(i=0,1)$ is the basis function that changed according to dimming level. Section 3 provides details of the basis function. After passing through a VPPM pulse-shaping filter, the transmitted signal has the following form:

$$
x(t)=\sum_{i=-\infty}^{\infty} s\left(t-i T_{\mathrm{d}}\right)
$$

where $T_{\mathrm{d}}$ is the total time duration required to transmit each data block, $T_{\mathrm{d}}=T_{\mathrm{s}}+T_{\mathrm{g}}$, where $T_{\mathrm{s}}$ is the symbol duration and $T_{\mathrm{g}}$ is the guard time to avoid inter-symbol interference caused by channel dispersion. The LED is driven by the current signal controlled by $x(t)$. The LED emits the light signal $X(t)$, which has the mean optical power, $P_{t}=\frac{1}{T} \int_{0}^{T_{\mathrm{d}}} X(t) d t$. After passing through the optical channel $h_{\mathrm{o}}(t)$, the optical signal $y(t)$ received is given as follows:

$$
y(t)=X(t) * h_{\mathrm{o}}(t)+n_{\mathrm{o}}(t)
$$

where $" *$ denotes convolution and $n_{\mathrm{o}}(t)$ is the optical noise source.

Subsequently, $y(t)$ is converged to an electric signal through a photodiode (PD) to produce the signal $r(t)$ as follows:

$$
\begin{aligned}
r(t) & =R \cdot y(t) \\
& =R\left(X(t) * h_{\mathrm{o}}(t)+n_{\mathrm{o}}(t)\right) \\
& =H(0) \cdot x(t) * h(t)+n(t),
\end{aligned}
$$

where $R$ is the PD conversion responsivity (A/W), $H(0)$ means the path loss gain of the signal, $h(t)$ denotes the electrical impulse response of the optical wireless channel, and $n(t)$ is the electrical additive white Gaussian noise.

This paper adopts the diffuse channel model to understand the effects of the channel condition in an operating VPPM scheme. By examining previous studies in optical wireless channel models [5-7], we propose the multiple exponential channel model given as

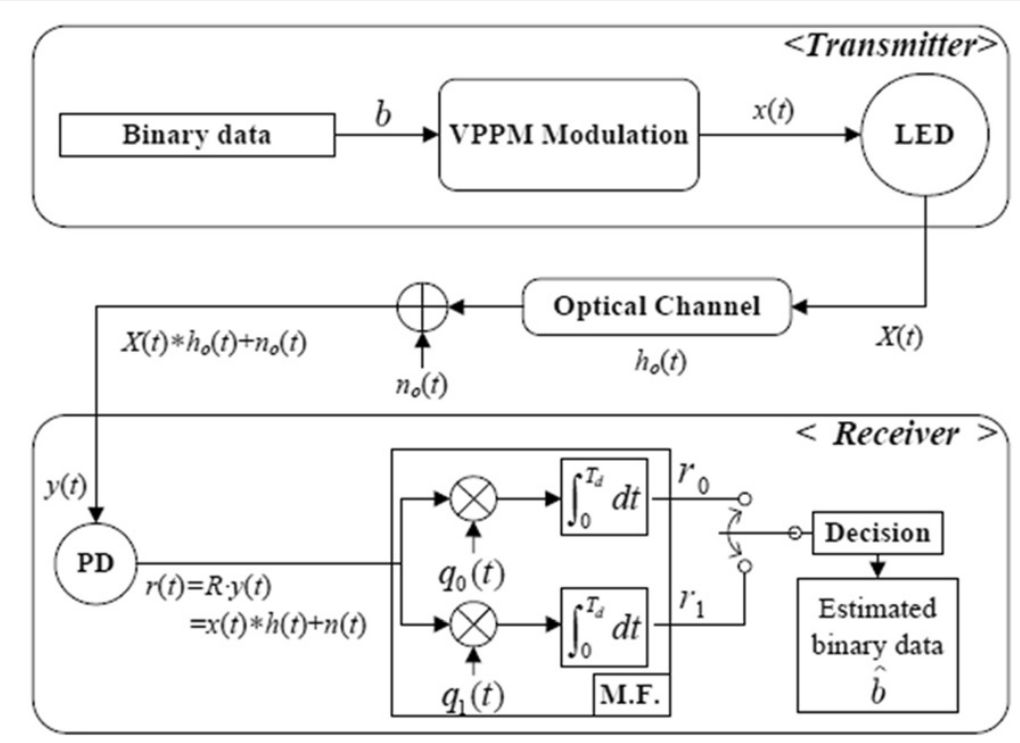

Figure $\mathbf{2}$ The proposed structure of VLC system based on VPPM. 


$$
h(t)=\sum_{i=0}^{C} G_{i} \cdot \exp \left(-\tau_{i} \cdot\left(t-t_{d, i}\right)\right) \cdot u(t),
$$

where $C$ is the number of channel cluster, $G_{i}$ is the channel gain of the $i$ th cluster, $\tau_{i}$ is the time constant of the $i$ th cluster, and $t_{d, i}$ is the time delay of the $i$ th cluster.

The hard decision decoding at the receiver was considered. The receiver was also assumed to be synchronized precisely with the transmitter. When $r(t)$ is received, the receiver will generate the received vector $r=\left[r_{0}, r_{1}\right]$ through a matched filtering process as follows:

$$
\begin{aligned}
& r_{0}=\int_{0}^{T_{\mathrm{d}}} r(t) \cdot q_{0}(t) d t \\
& r_{1}=\int_{0}^{T_{\mathrm{d}}} r(t) \cdot q_{1}(t) d t
\end{aligned}
$$

where $q_{i}(t)=\phi_{i}(t) * h(t)(i=0,1)$ denotes the template pulse dispersed by the channel.

Based on the maximum likelihood decision rule, the transmitted signal is detected as

$$
\hat{b}=\arg \max _{j=0,1} r_{j}
$$

\section{Performance analysis}

As mentioned previously, the error performance of VPPM for determining the performance tradeoff between dimming control and data communication according to the channel condition was derived. The basis function shown in (1) is defined as follows:

$$
\begin{aligned}
& \phi_{0}(t)=\left\{\begin{array}{cc}
\sqrt{\frac{100}{d \cdot T_{s}}}, & 0 \leq t \leq \frac{d \cdot T_{s}}{100} \\
0 & \text { otherwise }
\end{array}\right. \\
& \phi_{1}(t)=\left\{\begin{array}{cl}
\sqrt{\frac{100}{d \cdot T_{s}},}, & \left(1-\frac{d}{100}\right) \cdot T_{s} \leq t \leq T_{s} \\
0 & , \quad \text { otherwise }
\end{array}\right.
\end{aligned}
$$

where $\phi_{i}(t)$ and the corresponding template pulse $q_{i}(t)$ are normalized to have a unit energy as $\int_{0}^{T_{\mathrm{s}}} \phi_{i}^{2}(t) d t=1$ and $\int_{0}^{T_{\mathrm{s}}} q_{i}^{2}(t) d t=1$. Figure 3 shows the signaling structure of the basis function, $\phi_{i}(t)$, and the template pulse, $q_{i}(t)$.

If it is assumed that data bit ' 0 ' is transmitted $(b=0)$, the signal received is rewritten as follows:

$$
r(t)=\gamma \cdot \sqrt{E_{\mathrm{s}} \cdot \frac{d}{50}} \cdot q_{0}(t)+n(t)
$$

where $\gamma=R \cdot H(0)$ means the scaling coefficient that contains the effect of the channel path loss gain and PD responsivity.

After matched filtering, the received vector $\boldsymbol{r}$ can be obtained as follows:

$$
\begin{aligned}
r & =\left[r_{0}, r_{1}\right] \\
& =\left[\gamma \cdot \sqrt{E_{\mathrm{s}} \cdot \frac{d}{50}}+n_{0}, \gamma \cdot \sqrt{E_{\mathrm{s}} \cdot \frac{d}{50}} \cdot \alpha+n_{1}\right],
\end{aligned}
$$
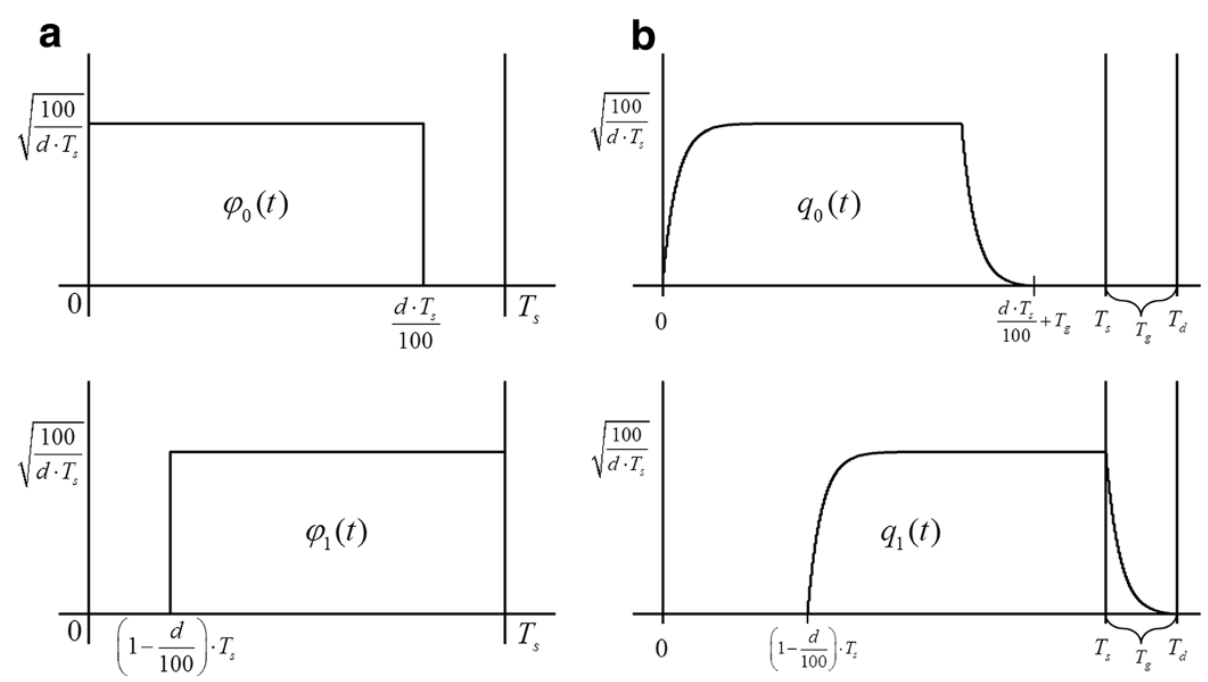

Figure 3 The signaling structure of (a) the basis function and (b) the template pulse. The number of cluster $(C)=1$. 
Table 1 Channel parameters for simulations

\begin{tabular}{lll}
\hline Channel & Parameter & Value \\
\hline LOS & $h(t)=\delta(t)$ & \\
Diffuse & Number of clusters & 1 \\
& Channel gain $\left(G_{j}\right)$ & 1 \\
& Time delay $t_{d, i}$ & $20 \mathrm{~ns}$ \\
& Time constant & $2.3 \times 10^{8}$ \\
\hline
\end{tabular}

$$
\begin{aligned}
r_{0} & =\int_{0}^{T_{\mathrm{d}}} r(t) \cdot q_{0}(t) d t \\
& =\int_{0}^{T_{\mathrm{d}}} \gamma \cdot \sqrt{E_{\mathrm{s}} \cdot \frac{d}{50}} \cdot q_{0}^{2}(t) d t+\int_{0}^{T_{\mathrm{d}}} q_{0}(t) \cdot n(t) d t \\
& =\gamma \cdot \sqrt{E_{\mathrm{s}} \cdot \frac{d}{50}}+n_{0} \\
r_{1} & =\int_{0}^{T_{\mathrm{d}}} r(t) \cdot q_{1}(t) d t \\
& =\int_{0}^{T_{\mathrm{d}}} \gamma \cdot \sqrt{E_{\mathrm{s}} \cdot \frac{d}{50}} \cdot q_{0} \cdot q_{1}(t) d t+\int_{0}^{T_{\mathrm{d}}} q_{1}(t) \cdot n(t) d t \\
& =\gamma \cdot \sqrt{E_{\mathrm{s}} \cdot \frac{d}{50}} \cdot \alpha+n_{1}
\end{aligned}
$$

where $n_{0}, n_{1}$ becomes Gaussian random noise with a zero mean and variance $N_{0} / 2 . \alpha$ represents the correlation factor, which is defined as follows:

$$
\alpha=\int_{0}^{T_{\mathrm{d}}} q_{0}(t) \cdot q_{1}(t) d t
$$

In addition, two noise terms $\left(n_{0}, n_{1}\right)$ were also correlated due to the correlation factor $\alpha$ as shown below:

$$
\begin{aligned}
E\left\{n_{0} \cdot n_{1}\right\} & =\int_{0}^{T_{\mathrm{d}}} \int_{0}^{T_{\mathrm{d}}} E\{n(t) n(\tau)\} \cdot q_{0}(t) \cdot q_{1}(\tau) d t d \tau \\
& =\frac{N_{0}}{2} \cdot \int_{0}^{T_{\mathrm{d}}} q_{0}(t) \cdot q_{1}(\tau) d t=\frac{N_{0}}{2} \cdot \alpha .
\end{aligned}
$$

Accordingly, it affects the performance of VPPM. Therefore, it is important to consider this correlation factor carefully when operating VPPM.

To evaluate the error performance analytically, the new random variable is defined as $z=r_{0}-r_{1}$. The conditional mean and variance of $z$ are given as follows:

$$
\begin{aligned}
& \begin{aligned}
E\{z \mid b & =0\}=E\left\{r_{0}\right\}-E\left\{r_{1}\right\} \\
& =\gamma \cdot \sqrt{E_{\mathrm{s}} \cdot \frac{d}{50}} \cdot(1-\alpha)
\end{aligned} \\
& \operatorname{Var}\{z \mid b=0\}=E\left\{\left(n_{0}-n_{1}\right)^{2}\right\}=N_{0} \cdot(1-\alpha) .
\end{aligned}
$$

Noting that the position with a maximum value among $\left\{r_{j}\right\}_{j=0}^{1}$ is determined to be the transmitted signal in the ML scheme, the error probability can be expressed as [8]

$$
P_{e \mid b=0}=P(z<0 \mid b=0)=\int_{-\infty}^{0} p_{z}(z \mid b=0) d z
$$

where

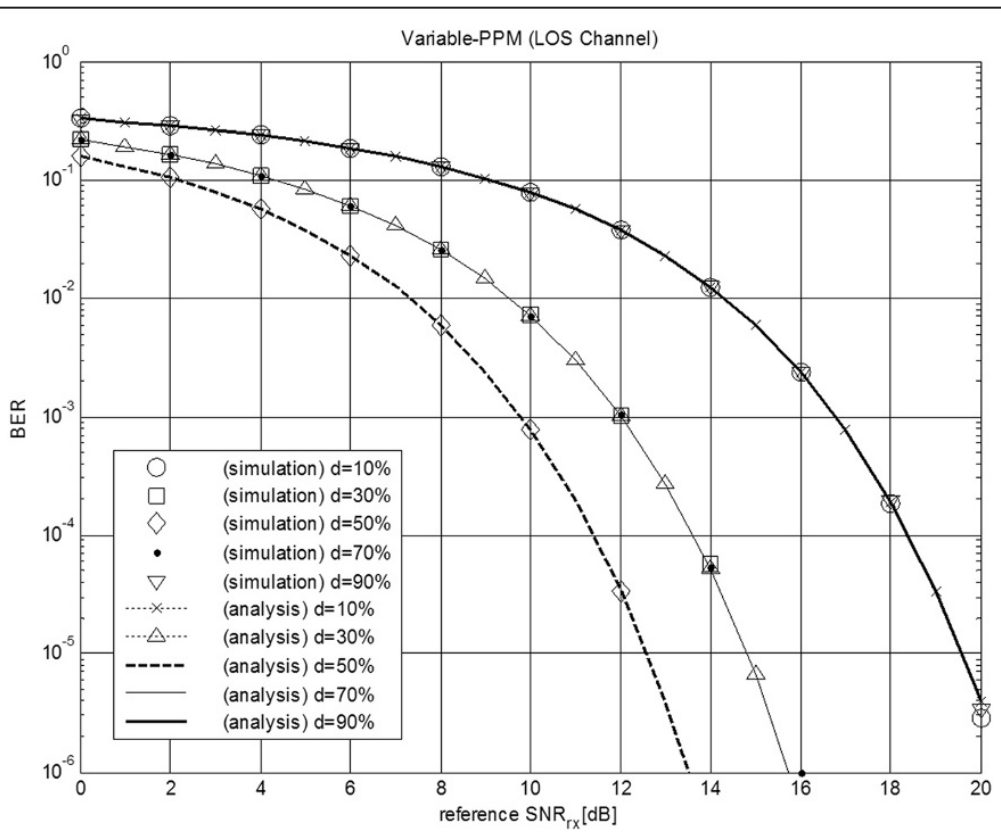

Figure 4 The BER performance of VPPM according to reference $S_{N R}$ in the LOS channel case. 

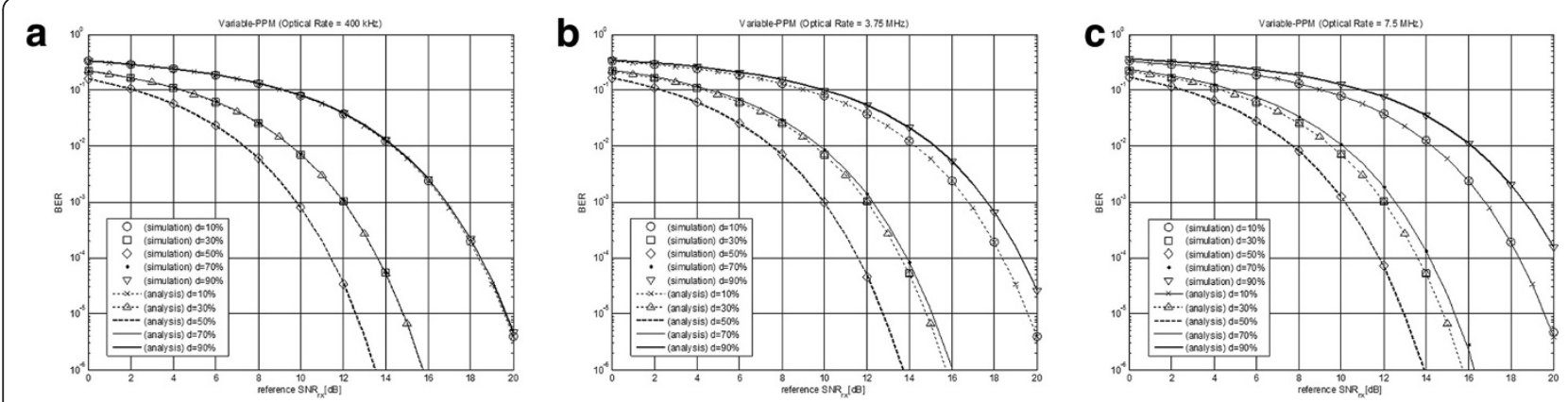

Figure 5 The BER performance of VPPM in a diffuse channel case according to optical rate of LED. (a) $400 \mathrm{kHz}$, (b) $3.75 \mathrm{MHz}$, and (c) $7.5 \mathrm{MHz}$.

$$
p_{z}(z \mid b=0)=\frac{1}{\sqrt{2 \pi N_{0}(1-\alpha)}} \exp \left[-\frac{\left(z-\gamma \cdot \sqrt{E_{\mathrm{s}} \cdot \frac{d}{50}} \cdot(1-\alpha)\right)^{2}}{2 N_{0}(1-\alpha)}\right] .
$$

Using (18) and (19), the error probability, $b=0$, can be derived as follows:

$$
P_{e \mid b=0}=\frac{1}{2} \operatorname{erf} c\left(\gamma \cdot \sqrt{\frac{E_{\mathrm{s}}}{2 N_{0}}} \cdot \sqrt{\frac{d \cdot(1-\alpha)}{50}}\right)
$$

where $\operatorname{erf} c(x)=\frac{2}{\sqrt{\pi}} \int_{x}^{\infty} \exp \left(-t^{2}\right) d t$. Similarly, the error probability, $P_{e \mid b=1}$, can be derived as follows:

$$
\operatorname{erf} c(x)=\frac{2}{\sqrt{\pi}} \int_{x}^{\infty} \exp \left(-t^{2}\right) d t
$$

Because $a$ priori probability of $b=0$ and $b=1$ is equal to $1 / 2$, the error probability of VPPM can be expressed as

$$
\begin{aligned}
P_{e} & =P\{b=0\} \cdot P_{e \mid b=0}+P\{b=1\} \cdot P_{e \mid b=1} \\
& =\frac{1}{2} \operatorname{erf} c\left(\gamma \cdot \sqrt{\frac{E_{\mathrm{s}}}{2 N_{0}}} \cdot \sqrt{\frac{d \cdot(1-\alpha)}{50}}\right) .
\end{aligned}
$$

\section{Simulation result}

The VPPM scheme was described, and its error performance was analyzed. This paper presents the analytical and simulated results to test the validity of the analysis and the relationship between the dimming and communication performance in the line-of-sight (LOS) and diffuse channel cases.

By referring to references [5-7], the channel parameters for the simulation are listed in Table 1.

Figure 4 shows the analytical and simulated bit error rate (BER) performance according to the reference received signal-to-noise ratio $\left(\mathrm{SNR}_{\mathrm{rx}}\right)$ by changing the dimming levels in the LOS channel case. The reference $\mathrm{SNR}_{\mathrm{rx}}$ denotes the amount of noise power by setting the signal power with a $50 \%$ dimming level as the reference signal power. Therefore, the actual $\mathrm{SNR}_{\mathrm{rx}}$ will be changed according to the dimming ratio. The figure shows that the analytical curves are well matched with the simulation curves. Therefore, the analytical results
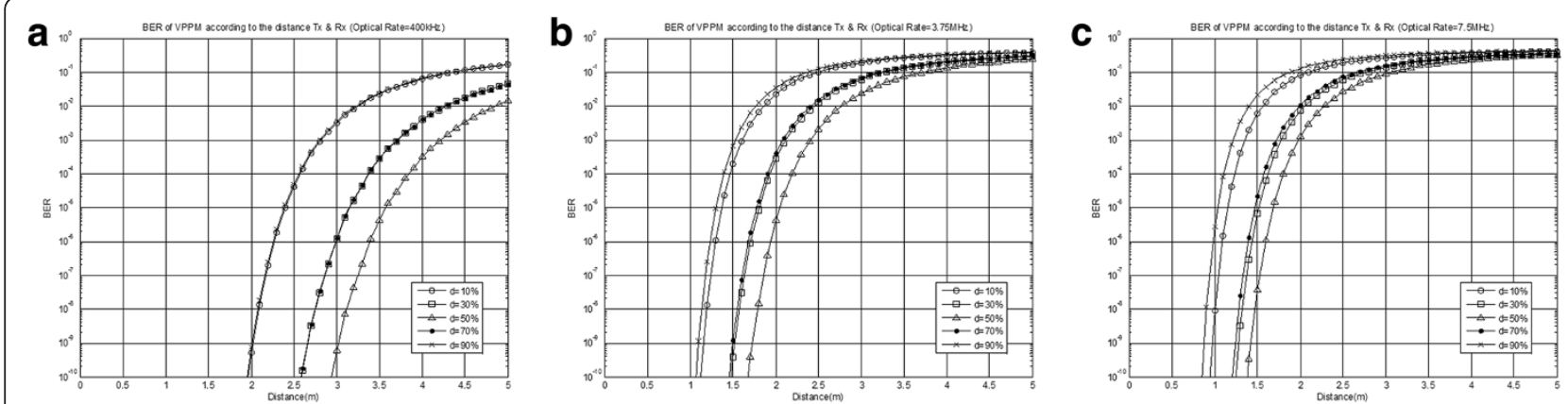

Figure 6 BER performance of VPPM. The performance is based on the distance between TX and RX, dimming ratio, and optical rate of LED when LED TX power is $0.1 \mu \mathrm{W}$. (a) optical rate $400 \mathrm{kHz}$, (b) optical rate $3.75 \mathrm{MHz}$, (c) optical rate $7.5 \mathrm{MHz}$. 
provide a good estimation of the performance when the system parameters have been selected. VPPM showed the best performance at the $50 \%$ dimming level with the BER degrading symmetrically as the dimming level strays from $50 \%$. A dimming level less than $50 \%$ is due to less allocated signal energy. When the dimming level is greater than $50 \%$, the increasing correlation factor degrades the performance faster than the increase in allocated signal energy. Therefore, the dimming levels in VPPM strongly affect the communication performance.

Figure 5 shows the analytical and simulated BER performance in the diffuse channel case. The optical rate of the LED according to the IEEE standard was changed to examine the effects of channel dispersion on the communication performance [4]. Figure 5a shows the result when the optical rate is $400 \mathrm{kHz}$. A similar result was obtained in the LOS case. On the other hand, the BER performance of the optical rate $(3.75$ and $7.5 \mathrm{MHz})$ in Figure $5 \mathrm{~b}, \mathrm{c}$ is degraded when the dimming level is greater than $50 \%$. This is because the relative length of the channel delay spread is becoming longer than the symbol duration $\left(T_{\mathrm{s}}\right)$ as the optical rate is increasing. This causes a larger correlation factor, which degrades the BER performance.

Figure 6 shows the relationship between VPPM and the BER performance according to (1) the distance between TX and RX, (2) the dimming ratio, and (3) the optical rate of a LED under the VLC scenario in reference [1]. Because the VLC performance based on VPPM affects the design of LED illumination infrastructure, it will be meaningful to express communication performance based on the distance, optical rate, and dimming ratio under a given VLC scenario. Therefore, the actual $\mathrm{SNR}_{\mathrm{rx}}$ will be determined as a function of the distance, dimming level, optical rate, and environment settings. Figure 6a shows that the distance between TX and RX should be in the range of approximately 2.5 to $3.8 \mathrm{~m}$ to achieve a $10^{-4}$ BER and $10 \%$ to $90 \%$ dimming flexibility. In the case of Figure $6 \mathrm{~b}, \mathrm{c}$, the distance between TX and RX becomes shorter to obtain the same performance (approximately $1.4 \sim 2.2 \mathrm{~m}$ at a $3.75-\mathrm{MHz}$ optical rate and $1.5 \sim 1.8 \mathrm{~m}$ at a $7.5-\mathrm{MHz}$ optical rate). This is because the noise bandwidth and correlation factor due to a channel dispersion increase with increasing optical rate.

\section{Conclusion}

The error performance of VPPM was analyzed, and the results were confirmed by a simulation under LOS and diffuse channel conditions. An examination of the relationships among the BER curve, dimming level, and optical rate according to the reference $\mathrm{SNR}_{\mathrm{rx}}$ revealed tradeoffs between the dimming flexibility and communication performance according to the channel condition if the VPPM scheme for VLC is considered. Care should be taken when designing LED illumination infrastructure with a VLC support based on VPPM because the $\mathrm{SNR}_{\mathrm{rx}}$ in a VLC is related to the LED luminance distribution.

\section{Competing interests}

The authors declare that they have no competing interests.

\section{Acknowledgments}

This research was supported by the Basic Science Research Program through the National Research Foundation of Korea (NRF), funded by the Ministry of Education, Science and Technology (No. 2010-0021118).

Received: 26 March 2012 Accepted: 8 May 2013

Published: 24 May 2013

References

1. T Komine, M Nakagawa, Fundamental analysis for visible-light communication system using LED lights. IEEE Trans On Consumer Electron 50(1), 100-107 (2004)

2. J Grubor, S Randel, K-D Langer, JW Walewski, Broadband information broadcasting using LED-based interior lighting. J Lightw Technol 26(24), 3883-3892 (2008)

3. T Komine, JH Lee, S Haruyama, M Nakagawa, Adaptive equalization system for visible light wireless communication utilizing multiple white LED lighting equipment. IEEE Trans Wireless Comm 8(6), 2892-2900 (2009)

4. IEEE Std 802.15.7, IEEE Standard for Local and Metropolitan Area NetworksPart 15.7, Short-Range Wireless Optical Communication Using Visible Light (IEEE, Piscataway, 2011)

5. JB Carruthers, P Kannan, Iterative site-based modeling for wireless infrared channels. IEEE Trans Antennas and Propagation 50(5), 759-765 (2002)

6. JR Barry, JM Kahn, WJ Krause, EA Lee, DG Messerschmitt, Simulation of multipath impulse response for indoor wireless optical channels. IEEE J Sel Area Comm 11(3), 367-379 (1993)

7. X Zhang, K Cui, M Yao, H Zhang, Z Xu, Experimental characterization of indoor visible light communication channels, in 8th International Symposium on Communication Systems, Networks \& Digital Signal Processing (CSNDSP) (Poznan, 2012). 8-20 July 2012, pp. 1-5

8. JG Proakis, M Salehi, Digital Communication, 4th edn. (McGraw-Hill, New York, 2006)

doi:10.1186/1687-1499-2013-134

Cite this article as: Yoo and Jung: Modeling and analysis of variable PPM for visible light communications. EURASIP Journal on Wireless Communications and Networking 2013 2013:134.

\section{Submit your manuscript to a SpringerOpen ${ }^{\circ}$ journal and benefit from:}

- Convenient online submission

- Rigorous peer review

- Immediate publication on acceptance

- Open access: articles freely available online

- High visibility within the field

- Retaining the copyright to your article

Submit your next manuscript at springeropen.com 